\title{
Quantifying Developing Country Research Capacity in the Areas of Malaria, Schistosomiasis, and Leprosy
}

\author{
Esther K. Hicks, Pepin G. Cabo, Floor Rikken
}

Implicit in addressing the issue of how to build and strengthen health related endogenous research capacity is that both donor organizations and developing country policymakers distinguish between health priorities and health research priorities. This distinction has important policy consequences in that the majority of developing countries are not in a position, either in financial or human resource terms, to simultaneously generate and implement broad-based priorities in both areas. In this context, it is important that health research priority setting be developed in tandem with existing research capacity. This underlines the importance of assessing the amount and nature of available expertise, and positioning research output, both in the national health needs, and the international science research contexts.

The present article, which more properly constitutes a feasibility study, uses scientometric methods to assess the amount and nature of scientific output in three important tropical diseases: malaria, schistosomiasis and leprosy. The major goals of this study have been to i) compare the amount and nature of research produced in developed and developing countries for the three diseases; ii) determine in how far scientometric methods can be used to measure research capacity, and whether this measurement can facilitate the determination of health (research) priorities.

\section{Introduction}

The issue of how to adequately define "possible and practicable" national health-related priorities in developing countries, and how to build and strengthen related endogenous research capacity has been of considerable concern, both to developing and donor country governments.

Esther K. Hicks is general secretary for the Advisory Council for Scientific Research in Development Problems in The Netherlands. She is also editor of Knowledge and Policy.

Pepin Cabo is a Ph.D. candidate in the department of management and organization, University of Groningen.

Floor Rikken is a Ph.D. candidate at the University Center for Pharmacology, University of Groningen. Address for correspondence: Faculty of Organization and Management, University of Groningen, P.O. Box 800, 9700 AV Groningen, The Netherlands. 
Implicit in addressing this issue is that both donor organizations and developing country policymakers distinguish between health priorities and health research priorities.

The former involves national social and health policy development designed to effect urgently required improvements in the social (to include health) and physical environment of the population concerned. The latter involves research into, for example, vaccine development, drug treatment, disease control and management.

This distinction has important policy consequences in that the majority of developing countries are not in a position, either in financial or human resource terms, to simultaneously generate and implement broad-based priorities in both areas. Thus, it is important that health research priority setting in, for example, biomedical research on infectious and parasitic diseases, be designed to meet national health needs.

Moreover, health research priority setting must be seen in tandem with existing research capacity. Implicit here is the question of how to adequately define "possible and practicable" national health-related priorities, and how to build and strengthen related endogenous research capacity.

In this context, we have presumed that the overall objective to build endogenous research capacity is based on the assumption that a strong science and technology foundation is important for high-level training and the development of essential applications (as well as for their evaluation). However, the relatively low research expenditures of developing countries underlines the importance of making appropriate strategic research choices. This enhances the need for comprehensive, well-structured information on the existing science base (in input and output terms). Ideally, this involves the systematic assessment of such aspects of science as the structure and development of scientific fields, S\&T interaction, research collaboration (national and international), research performance (quantity and quality), to name but a few (Van Raan, 1993:2).

Thus, it is important from the perspective of both donor organizations and developing country governments, that inventories and assessments be made of the amount and nature of available expertise in the health research sector in developing countries. Moreover, that this research output is positioned in the global and national health-needs context. This is especially important in light of the general assumption that research capacity is limited and output is low in most developing countries (even in the best universities) (see, for example, Thulstrup, 1992:4; Gaillard, 1990:348).

The increasing need to assess the many aspects of scientific activity in an organized and structured manner has generated the development and refinement of scientometrics as a quantitative tool used to support and supplement traditional qualitative peer review.

As such, it is a valuable tool to use in conjunction with the objective to build endogenous research capacity. It can be used to generate inventories and assessments of the amount and nature of available expertise in, for example, the health research sector in developing countries. 
In addition, scientometric analyses can be used to determine the size and characteristics of scientific impact and the structural features of science.

Nevertheless, the present study, which has been limited to three specific health research sectors, is concerned only to determine the size and characteristics of (global) scientific output in three important tropical diseases: malaria, schistosomiasis, and leprosy (see sidebars).

There were two primary reasons for this limitation: the assumption that biomedical research in infectious and parasitic diseases will continue to constitute a priority in many developing countries; and at the outset we were unclear about the degree to which developing countries produced more than a negligible amount of research in any health sector.

Consequently, the following more properly constitutes a feasibility study and, as such, cannot provide information about the scientific or health policyrelated impact of the knowledge produced in these three research areas. It must also be stressed that while high priority continues to be given to biomedical research on such diseases, their actual prevention (and to some extent control) is primarily a sociopolitical issue.

Accordingly, the major goals of the present study have been to 1) compare the amount and nature of research produced in developed and developing countries for the three diseases and; 2) determine how far scientometric methods can be used to measure research capacity, and whether this measurement can facilitate the determination of health (research) priorities.

The areas of malaria, schistosomiasis and leprosy were chosen for four primary reasons:

- Not only do malaria, schistosomiasis and leprosy constitute widespread tropical diseases, but all three strongly influence the pattern of illness in many developing countries. As a result, research in these areas has strong support both among donor country governments and in developing countries where such diseases are endemic.

- Because research in these areas is generally of a biomedical nature, we presumed that researchers and related institutions must compete for funding on the international market, and/or must participate in international peer review. At both the national and international level, such research is normally evaluated in science intrinsic (i.e., output) terms.

- Other areas of health research (e.g., primary health care) either proved too difficult to circumscribe for efficient quantitative analyses, or the volume of research produced in such areas is often "grey," and is not always included in international data bases. ${ }^{1}$

- We assumed the existence of some measurable output from developing countries where such diseases are endemic. This assumption was made in conjunction with, for example, the (in 1976 W.H.O. initiated) TDR program goal of strengthening biomedical research capacity in the tropical countries where these diseases are endemic (RAWOO Advisory Report on Health and Illness in Developing Countries, 1984:1-2).

This article begins with a discussion of the distinction between health priorities and health research priorities, and the relationship between de- 
veloping such priorities and building and strengthening health research capacity in developing countries. Subsequently, an outline is presented of the method used to inventory the amount and nature of global biomedical research output for the three tropical diseases Malaria, Schistosomiasis and leprosy. Finally, consideration is given to the question of whether health research in developing countries should be primarily science led, or address the issue of disease control and prevention.

\section{Health and Health Research Priorities}

The economic crisis of the 1980s did not bode well for the health sector in developing countries. This has resulted in, for example, the postponement of extensions in primary health care; an inability to replace and repair equipment and facilities; severe cuts in the drug and manpower budget, to name but a few. Consequently, the setting of health-related priorities for most developing country governments (especially the poorest of these) is an almost insurmountable task. Of course, health-related priorities are also set by donor governments.

In both cases (i.e., developing and donor country governments), health priorities reflect political pressures and funding allocation, which, in turn, determine sector prioritization (i.e., the degree of support extended to basic research, targeted research, health care delivery services, social science research, health technology research, and so on).

This raises the two important, interrelated questions of how to adequately define "possible and practicable" national health-related priorities, ${ }^{2}$ and how to build and strengthen related endogenous research capacity.

For example, for some years now biomedical and clinical research has been a priority among both donor and developing country governments. This is reflected in, for instance, the fact that biomedical and clinical research capacity in developing countries tends to be somewhat stronger than in the areas of epidemiology, demography, and the social and behavioral sciences (ENHR report, 1991).

Of course, this is hardly surprising, given that the major cause of death and chronic illness in developing countries continues to be infectious and parasitic diseases (the most important of which include diarrheal diseases, tuberculosis, acute respiratory diseases, malaria, schistosomiasis). ${ }^{3}$ As a result, there has been a general consensus that biomedical science must continue to be strongly supported. Resources permitting, developing countries also strongly support such research at the national level.

Nevertheless, the validity of assigning a priority to biomedical research is disputable, considering that the pressing issues of the current rate of infection, and the increasing number of those at risk for infectious and parasitic diseases, are directly related to socioeconomic, political, and socioenvironmentally generated problems.

It is precisely in such a context that a distinguish between health priorities and health research priorities must be made.

Moreover, given that the majority of countries where such diseases are endemic are usually not in a position to systematically operationalize even 
"possible and practicable" biomedical and clinical research priorities (both in financial and human resource terms), the simultaneous assignment of health and health research priorities may actually undermine even the moderate potential success of both.

\section{Building and Strengthening Health Research Capacity}

Adding to the problems most developing countries encounter in generating (and implementing) national, social, environmental, and health care policy measures, has been a general incapacity to mount an adequate overall research effort. This has been attributed to such factors as institutional defects, poor logistical support, insufficient numbers of trained researchers, funding limitations, and so on (see, for example, ENHR, 1991:29).

The problem has been exacerbated by the ideal that each developing country should develop "the research capacity needed to analyze continually its health situation and to identify its major health problems; to respond to new challenges; and to develop better interventions for prevention, control and treatment" (ENHR, 1991:29).

To a great extent, this expectation has its basis in the fact that donor governments and agencies have invariably viewed all forms of capacity building in developing countries as part of the wider concern about the management of the economies of those countries. As such, donor organizations have regularly stressed the need for human resource development, institution building, and a reorientation of education and training. All of these strategies are (theoretically) designed to improve quality, relevance (social, health, etc.), and cost-effectiveness. Operationalizing such goals has been quite another matter, however.

A major problem, as Girwood has rightly pointed out, is the lack of clarity about what should be the nature of capacity building:

for the term 'capacity building' to be meaningful, clear expression must be given to the capacities which are to be enhanced or otherwise developed, for what purpose, and whether this will be at the level of the nation, the region, or even the continent as a whole. It would seem that some clearly-stated strategic principle must underlie the concept, and if this is lacking, then the term itself is somewhat vacuous. An element of strategic choice is always implicit, and particularly so within the current context of constrained resources (Girdwood, 1991:104)

This problem has also been evident in attempts to build health research capacity in developing countries.

Ideally, any move in this direction-especially in conjunction with developing national health research priorities, presumes that 1) a range of suitable actions appropriate to health needs has already been identified (if this is not the case then research and development are a prerequisite); and 2) that a determination has been made about the nature of funding allocation (i.e., whether relevant funding agencies are able to extend support to 
problem-led or science-led research, or whether support for some combination thereof is feasible).

We could expect to find that, in developing countries, research geared to national health needs has more usually been problem- than science-led. This is related to the fact that solutions to health issues may either not be available, be too costly to implement, or the requisite infrastructure and manpower may be insufficient for the task.

Of course, one of the first steps in attempting to identify possible methods to prevent ill health and death is to analyze the causes. It is primarily for this reason that the importance of biomedical science to the developing world has never been contended. However, this raises the related questions of where and by whom such fundamental research can best be carried out, and how its results are to be used, applied and implemented. In this context, it can cogently be argued that two factors are of importance in this decision-making process:

1) A major criterion must be that biomedical research-in one form or other, meet national health needs. This is because such research can produce tools, which, while valuable for further research, may have little value for disease control.

Moreover, while the broad objectives of such research may be clear enough, it is extremely difficult to link specific expenditures with health outcomes. Williams et al. list four fundamental problems in this respect: 1) scientific progress is difficult to predict; 2 ) we do not fully understand the effects of (inter)national and private program expenditure on scientific activity; 3 ) it is always difficult to transform scientific progress into medical practice, let alone into improvements in health; and 4) policy analysis has the difficult task of assigning social value to improved health and longevity (Williams et.al, 1976:v).

2) Only those health research components for which a high degree of expertise is already available should be included in national health research priority setting. This implies the need to inventory and assess the amount and nature of (national) expertise already available.

It is unfortunate that the accent on research capacity building attempts has traditionally been on strengthening major inputs into university research (e.g., physical facilities and human resources). Few attempts have been made by donor organizations to stimulate the systematic inventory and assessment of the quantity and quality of existing capacity (i.e., research and research training output) in developing countries. This has been based on the premise that research output is difficult to measure (Thulstrup, 1992:13). ${ }^{4}$

\section{Method}

In order to comparatively position developing country research capacity in the international context, the total productivity was inventoried in three (developing country related) medical research priority areas: malaria, schistosomiasis, and leprosy. 
The medical data base MedLine, CD-ROM version, was used to compile as complete as possible an international (journal article) publication listing for the period 1983-1991. This was based on a free text search, CD-ROM Silverplatter in the categories: "Malaria" (or "Blackwater Fever"), "Schistosomiasis" (or "Bilharziasis"), "Leprosy"). ${ }^{5}$ The subsequent data base analysis, which covered the period 1989, 1990, 1991, provided information about the geographical and institutional publication output. In conjunction with the limitations of MedLine entries, the search was restricted to the period 1989, 1990, 1991 (i.e., the indication of addresses in MedLine is a recent phenomenon). In 1991, 85 percent of the records indicate an address; 82 percent in 1990; 62 percent in 1989; and 59 percent in 1988.

The primary focus of the search was on the north/south distribution of articles in forefront journals. Not only do the latter constitute the major media in the science-knowledge production system, but they can be distinguished according to regional/international and specific/general character. Additionally, specific research fields can often be easily identified by the nature of individual journals. This facilitated a general thematic categorization of research output, specified by a subsequent key-word analysis enabling the cognitive characterization of research fields. Both analyses (journal and keyword) were conducted for the period 1983, 1985, 1987, 1989, 1991. Finally, a comparative analysis was made for developed and developing countries of the most frequently occurring key words for the composite period 1989, 1990, and 1991.

The goal of the analyses was to determine the thematic specialization of individual countries. This was accomplished by identifying the correspondence between countries and journals for the period 1989-1991; and, identifying the correspondence between countries and key-words for the same period (it must be noted, however, that a major limitation of this type of bibliometric study is that it precludes a broad-based (to include sub-fields) comparative analysis).

With respect to the three medical research priority

\section{Malaria}

Malaria constitutes a major public health problem in the majority of tropical regions. Of the total world population, 9 percent $(474$ million people) inhabit areas where malaria is endemic. Some 300 million people are believed to be infected, 90 percent of which live in tropical Africa (W.H.O., 1993a:15). Outside of Africa, cases annually reported to W.H.O. are concentrated in nine countries: Afghanistan, Brazil, China, India, Mexico, the Philippines, Sri Lanka, Thailand and Vietnam.

The transmission of malaria involves a complex variety of factors (technical, socio-cultural and economic). Examples of the technical problems that continue to prevail include the drug resistance of parasites; vector resistance to insecticides; the various factors associated with human ecology; administrative and financial obstacles with respect to the implementation of effective control programmes. Other factors include poverty, precarious living conditions, agricultural and industrial expansion, migration, an inefficient (or lacking) health infrastructure, to name but a few. 
areas, at the outset we were interested in the following interrelated factors:

- How the number and nature of developing country productivity over time (in so far as it appears in the international science indicator database listing used), compares to that produced elsewhere (in developed and other developing countries).

- Determining the amount, nature (e.g., fundamental, applied, clinical), and output patterns of the global research contribution in order to conduct a comparative thematic analysis. This has potential use as an indicator of research related strengths and weaknesses in the context of national (where these were known), and international health research agendas.

- Identify and compare the distribution of output, both at the national and the international level. We presumed, for example, that a broad national distribution (institutionally speaking) of research productivity was indicative of strong endogenous research capacity. Moreover, identifying distribution patterns would enable both an assessment of the dissemination of information and an identification of research overlap and/or duplication.

\section{Results}

\section{Leprosy}

Leprosy is a chronic communicable disease caused by the Mycobacterium leprae, which is closely related to $M$. tuberculosis (the cause of tuberculosis). The only known reservoir of the former, which has an affinity for nerves and skin, is the human. The disease, which is associated with a wide spectrum of clinical states, can be severe and disfiguring.

Although the means of infection and transmission are unclear, it is assumed that the leprosy bacillus enters the body by means of the respiratory tract, and is transmitted by nasal discharge or broken skin. No specific environmental factors have been associated with the distribution or increase in the occurence of leprosy.

The actual number of leprosy cases is not known, however, current worldwide estimates place prevalence at 10-12 million cases. It is found mainly in Asia, Africa and Latin America.

Although the disease continues to be a major public health problem, recent years have witnessed improvement in the clinical profile. Unfortunately, drug resistance continues to be a serious problem. 
in leprosy research. It can also claim an increasing share of the publications on malaria. Egypt, and to a lesser degree Brazil, are active producers in the area of schistosomiasis. Apart from the increase in research on malaria in India, and on schistosomiasis in Egypt, we found no evidence of significant shifts in the ranking of countries between 1989 and 1991.

In addition, although we did not control for the physical (institutional and geographic) distribution of output in developed countries, we did do so for the developing countries included in the top 5 percent of producers for the three research areas. Although the present analysis indicated that research productivity in these countries is broadly distributed, both geographically and institutionally, a more extensive analysis is necessary to determine formal research networks (on both the national and international levels).

The percentages of country shares indicates a structural difference between leprosy and the two other fields. In the former case, scientific output is much more concentrated in one country (i.e., India). In fact, India and the United States account for approximatively 50 percent of all output on leprosy.

Table 1 lists the total number of publications for the three research areas from those countries contributing at least 5 percent of the total articles published (for the years 1987, $1989,1990,1991)$.

\section{Schistosomiasis}

Schistosomiasis is a group of diseases caused by flat worms of the genus schistosoma (sometimes also called bilharziasis). The intermediate host of these worms is the acquatic snail, from whence it is transmitted to a human host. Of the sixteen species of schistosomes, the three known to infect humans are $\mathrm{S}$. haematobium (causing urinary schistosomiasis); S. mansoni and S. japonicum (both causing intestinal schistosomiasis).

Schistosomiasis is generally considered a chronic disease, with mortality rates generally under 1 percent (although this can reach higher levels in Egypt).

This disease has long been considered a major health problem in areas where water resources are being developed (e.g., in small reservoirs and irrigation systems). Moreover, because its control requires a long-term commitment, the overall success rate of planning directed toward surveillance and control is directly related to a country's level of socioeconomic development.

Schistosomiasis is one of the most widespread parasitic infections affecting human beings, and it is estimated that its prevalence is growing (primarily in conjunction with population migration). In 1991, the W.H.O. Expert Committee on the Control of Schistosomiasis (W.H.O., 1993:13) estimated that, globally, 200 million of the total 600 million people at risk were infected with schistosomiasis. The disease is now endemic in 76 countries and territories (including Africa, Latin America, Eastern Mediterranean (to include Turkey), Southeast Asia, the western Pacific) (W.H.O., 1990:27; W.H.O., 1993:14). The highest infection rates are found in Brazil, Egypt, Ghana and the Sudan. 
FIGURE 1

Total Publications, Schistosomiasis, Leprosy, and Malaria

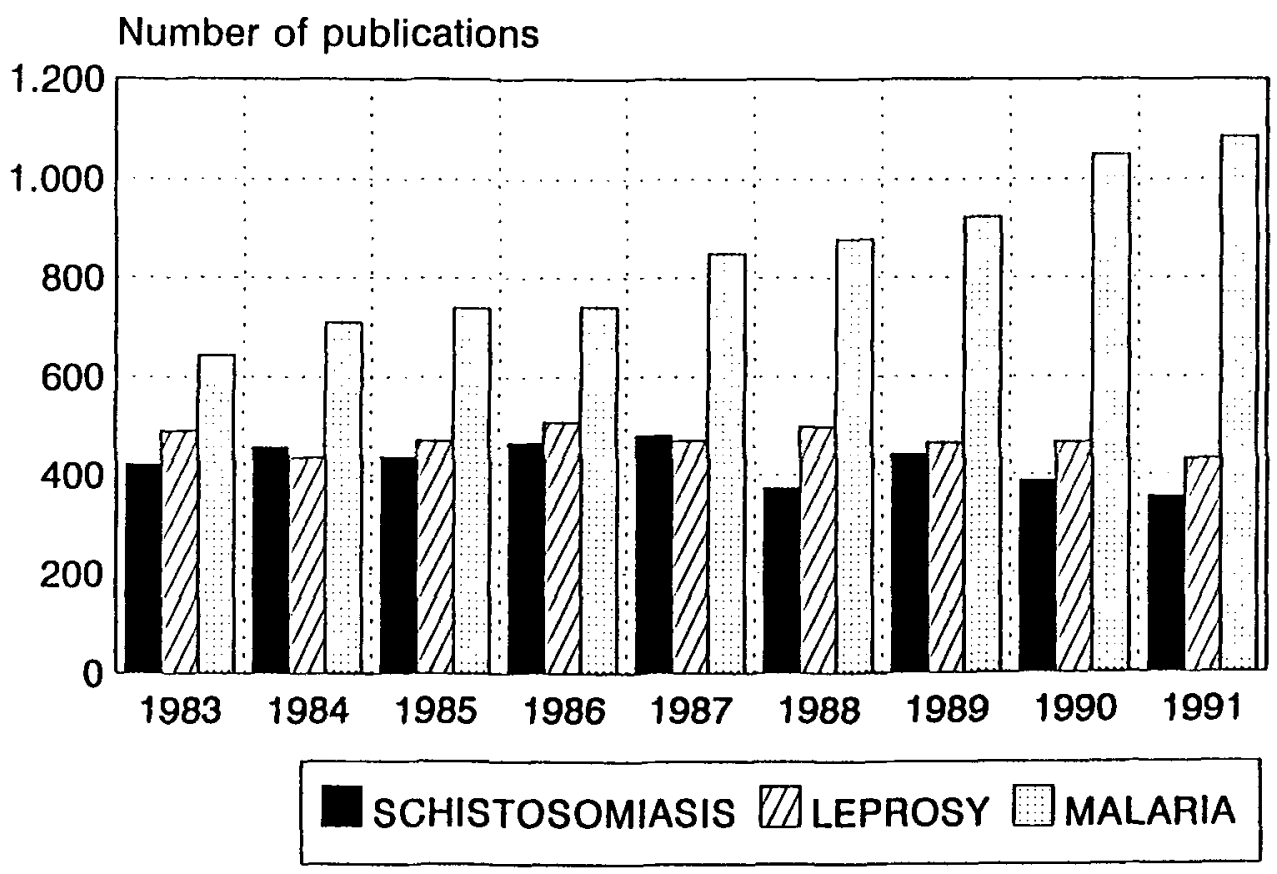

Source: MedLine

Figure 2 indicates the comparative distribution (according to total number of articles produced), by research area, for developing and developed countries. It can be seen that the share of articles produced by developing countries is quite substantial: approximately 50 percent in the fields of leprosy and schistosomiasis, and approximately 40 percent in the field of malaria (based on $>1 \%$ countries).

An analysis was made of the percentages of articles appearing in the top three journals (based on the top ten journals for every second year for the period 1983-1991). With the exception of schistosomiasis, the set of top journals remained constant during the time frame studied (expressed according to the number of different journals included in the top three between 1983-1991: for malaria, 4; for leprosy, 3; and for schistosomiasis, 8).

This configuration raised the question of how to compare the three research areas in terms of prominent journals. In this context, two dimensions seemed relevant: how international is the journal, and how specific (opposed to general) is the subject matter of the journal. ${ }^{6}$

The results of combining these two dimensions and scoring the journals indicted that, generally speaking, the structure for malaria output could be characterized "international"; leprosy as "specific"; and schistosomiasis as "international/specific." 
TABLE 1

Number of Publications (in percentile) of Those Countries Contributing at Least 5 Percent of Total Articles Published for Malaria for the Period 1989, 1990, 1991

\begin{tabular}{lrlrllr}
\hline & 1989 & & & & & 1991 \\
\hline USA & $27 \%$ & USA & & $19 \%$ & USA & $19 \%$ \\
UK & $13 \%$ & UK & & $10 \%$ & India & $10 \%$ \\
France & $6 \%$ & India & & $10 \%$ & France & $8 \%$ \\
India & $5 \%$ & France & $9 \%$ & UK & $8 \%$ \\
& & & & & Thailand & $6 \%$ \\
& & & & & Germany & $5 \%$ \\
\hline
\end{tabular}

TABLE 2

Number of Publications (in percentile) of Those Countries Contributing at Least 5 Percent of Total Articles Published for Leprosy for the Period 1989, 1990, 1991

\begin{tabular}{lrlrllll}
\hline & 1989 & \multicolumn{7}{c}{1990} & & & 1991 \\
\hline India & $32 \%$ & India & & $35 \%$ & India & $30 \%$ \\
USA & $18 \%$ & USA & & $16 \%$ & USA & $17 \%$ \\
UK & $10 \%$ & UK & & $8 \%$ & UK & & $10 \%$ \\
France & $5 \%$ & & & & & & \\
Brazil & $5 \%$ & & & & & \\
\hline
\end{tabular}

TABLE 3

Number of Publications (in percentile) of Those Countries Contributing at Least 5 Percent of Total Articles Published for Schistosomiasis for the Period 1989, 1990, 1991

\begin{tabular}{|c|c|c|c|c|c|}
\hline \multicolumn{2}{|c|}{1989} & \multicolumn{2}{|c|}{1990} & \multicolumn{2}{|c|}{1991} \\
\hline USA & $21 \%$ & USA & $15 \%$ & USA & $20 \%$ \\
\hline Brazil & $12 \%$ & Egypt & $14 \%$ & Egypt & $12 \%$ \\
\hline UK & $10 \%$ & UK & $10 \%$ & China & $9 \%$ \\
\hline Egypt & $7 \%$ & Brazil & $9 \%$ & Brazil & $6 \%$ \\
\hline France & $6 \%$ & France & $7 \%$ & UK & $6 \%$ \\
\hline & & Germany & $6 \%$ & France & $5 \%$ \\
\hline
\end{tabular}


FIGURE 2

Distribution of Total Number of Publications

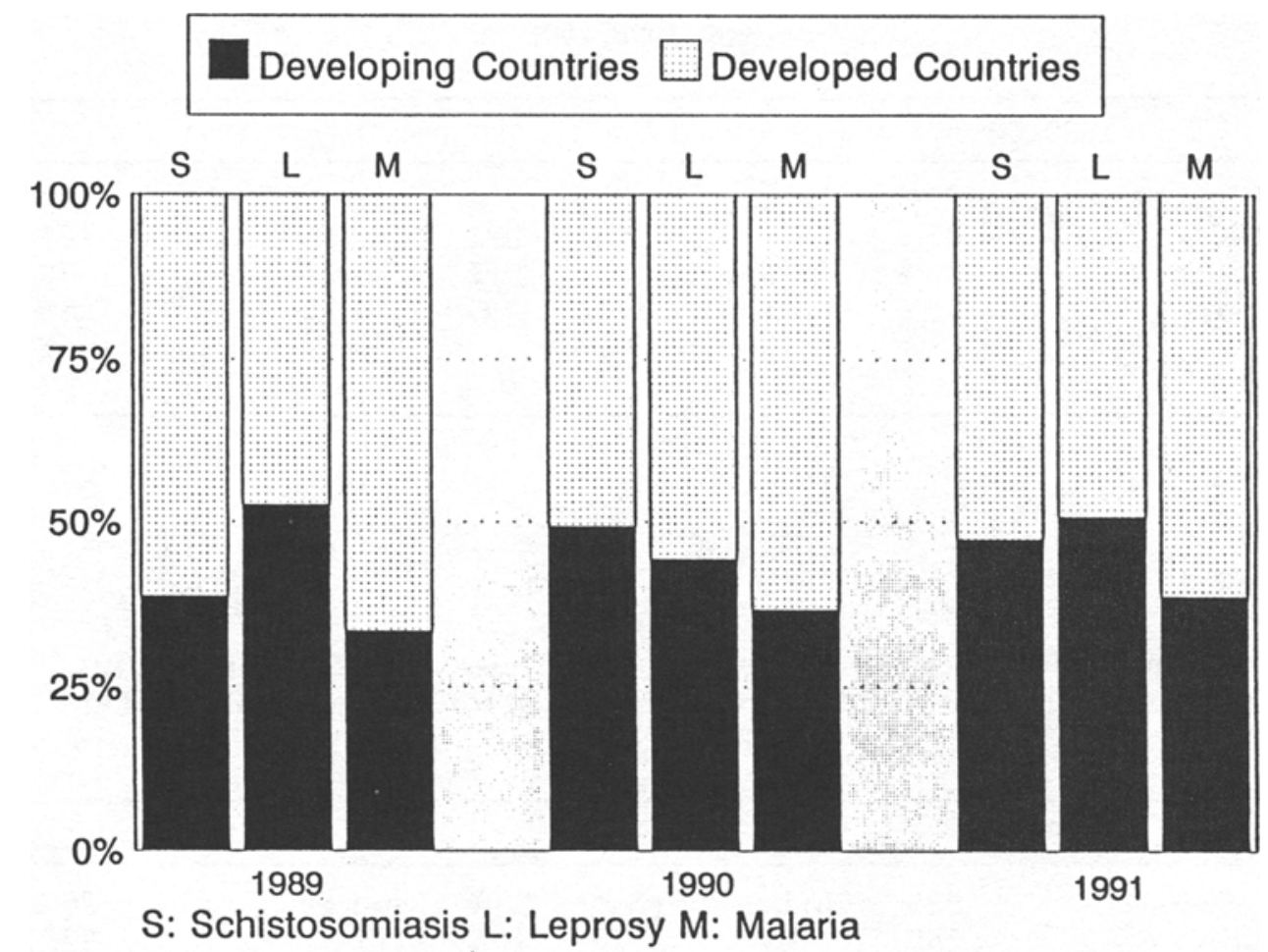

Subsequently, for each research area, the two top developing and the two top developed countries were used to determine the forefront journals for the period 1989, 1990, and 1991. Here, we were interested in determining whether research from all four countries was published in the same journals; or, if differences existed between developing and developed countries; and if the answer to these questions was different for each of the three diseases.

The analysis indicated that for each country publications are highly concentrated in one journal. For instance, in the United States, 12 percent of malaria and 15 percent of schistosomiasis publications appear in the American Journal of Tropical Medicine and Hygiene. In India, 32 percent of the output on malaria appears in the Indian Journal of Malariology. In Egypt, 40 percent of the output on schistosomiasis appears in the Journal of the Egyptian Society for Parasitology.

The analysis also indicated that the degree of journal specialization is higher for developed than for developing countries. Moreover, that the overwhelming trend in developing countries is to publish in local journals.

Further, that while publications from developing countries appear in "northern" journals, the converse is not the case (i.e., publications emanating from developed countries rarely appear in "southern" journals). An interesting exception to the latter trend is in the area of leprosy. Both U.S.- 
and U.K.-based researchers publish in the Indian Journal of Leprosy. It is, of course, possible that these researchers (i.e., in the case of leprosy) are of Indian origin and hold dual or honorary research appointments in their country of origin. However, a more plausible explanation can be found in the fact that India has, for some considerable time, had a strong tradition in fundamental research in a number of disciplines.

This was substantiated to some degree by the keyword analysis. For leprosy, there was generally more preclinical experimental research productivity in developing countries than in the areas of malaria and schistosomiasis (see below 'Leprosy').

Finally, an analysis of the differences in journal specialization according to research theme indicated that the vast majority of research produced in the developed world in all three areas was mainly of a pre-clinical experimental nature (for the period studied).

Subsequently, the same records used to determine the top journals were searched for the thirty most frequently appearing keywords (Medical Subject Headings), for the period 1989-1991. Using the Mesh, an analysis was made, per research area, of predominant key words. The search was conducted using all publications listed for the top 1 percent producers identified within the three research areas. ${ }^{7}$

The objective of this search was to ascertain whether the general nature and range of research output (e.g., preclinical (experimental); clinical) was similar for developed and developing countries.

Specific keywords were used to operationalize the distinction pre-clinical (experimental) and clinical (for example, words such as mice, antigens, antibodies, DNA, molecular sequence data indicated preclinical research; and words such as drug therapy, use, effects, resistance, and diagnosis, epidemiology, complications, prevalence indicated clinical research).

It was presumed that, in order for keywords to be an adequate indicator of preclinical and clinical research, the various operationalizations should give comparable results. This proved the case with respect to preclinical research (based on those keywords occurring in all three research areas). This could not, however, be done for clinical research: this category was absent from one of the groups (i.e., developed countries) in one research area (schistosomiasis), and only minimally represented in another (i.e., malaria).

The following results were found per research area:

Malaria: Based on a comparison of keywords indicating preclinical experimental research we found that, comparatively speaking, developed country-based publications proved more preclinical (experimental) in nature than those from developing countries. Substantially less such research (50 percent or less) was produced in developing countries (and in this case, no developing country research was found for "genetics-related" research).

Although developed countries did produce some research on drug therapy and effects, our indicators showed that developing countries led in drug-related research for the time frame considered. In contrast, only developed countries listed research on vaccine development. 
While some epidemiological research was listed for developed countries, the vast majority of clinically oriented research was conducted in developing countries.

Schistosomiasis: Here again, comparatively speaking, developed countrybased publications proved more preclinical (experimental) in nature than those from developing countries. Developing country productivity in this area was generally less than 50 percent of that produced by developed countries. As in the case of malaria, this was determined on the basis of a comparison of keywords indicating preclinical (experimental) research.

For the time frame considered, our indicators showed that research on drug therapy was only slightly more prevalent in developed countries. In contrast, clinical research was solely evident in developing countries.

Leprosy: Also in this case we found that, comparatively speaking, developed country-based publications proved more preclinical (experimental) in nature than those from developing countries. However, developing country productivity in this area was greater than that found in the other two research areas. With the exception of genetics-related research, which was restricted to the developed country group, preclinical research was generally at least half that produced in developed countries.

Although developed countries produced some research on drug therapy, our indicators showed that developing countries led in drug-related research.

Also in the case of clinical research, leprosy proved an exception. Here the groups "developed" and "developing" countries were roughly equal in the relative importance of those keywords indicating clinical research. However, developed country productivity was more in the area of diagnostic techniques, while research in developing countries was heavily concentrated in the areas of clinical diagnosis and control.

\section{Discussion}

The two interrelated goals of this study have been to compare the amount and nature of research produced in developed and developing countries for the three diseases and determine in how far scientometric methods can be used to measure research capacity, and whether this measurement can facilitate the determination of health (research) priorities.

The results of this study have indicated that the numerical share of articles published by the top producing developing countries is quite substantial in all three fields. Moreover, the research productivity within these countries is broadly distributed, both geographically and institutionally. A more extensive citation-based scientometric analysis would be necessary to determine research networks (on both the national and international levels).

Research production in developed countries was primarily preclinical in nature, whereas the majority of research produced in developing countries 
was of a more general nature (clinical and pathological, drug-related, and epidemiological).

This may be one reason why the majority of developing country research output is published in national or local journals. It may also explain why journal specialization occurs far less frequently in developing countries. Comparatively speaking, given the small amount of preclinical research produced, it can be expected that the majority of developing country scientists conducting such research will try to publish their results in specialized international journals, rather than those available at the national or local level. Additionally, the nature of the majority of developed country publications, and the journals in which these are published, may explain why few researchers from the developed world publish in "southern" journals. It may even be the underlying reason why north/south research collaboration (of equal partners) has been negligible (with the possible exception of the research area leprosy). ${ }^{8}$

On the other hand, the general nature of research in developing countries, and the fact that the majority of research output is published in national or local journals could reflect more fundamental problems; for example, a lack of facilities and funding requisite to conducting preclinical biomedical research, localized relevance, poor or variable research quality, or insufficient training and foreign language capacity.

The result of a combination of one or more of these factors with research dissemination limitations will not only considerably restrict accessibility for researchers in other countries where such diseases are endemic, but may engender (and propagate) research duplication. For example, in the case of malaria, both India and Thailand showed a substantial number of publications on drug application and evaluation (clinical and epidemiological); and for schistosomiasis an important research area in both Egypt and Brazil was drug therapy.

Conversely, it can also be said that research in developed countries shows reasonable consistency, both with respect to its general nature (more preclinical), and the journals in which results are published. However, it is in the developing countries where these diseases are endemic, and where governments have (often severely) delimited resources. Consequently, it is precisely these countries that can ill afford research not directly related to the control and prevention of endemic diseases.

This brings us to two interrelated questions:

1. Where and by whom can fundamental biomedical research in the three disease areas under study best be carried out, and how should the results be used, applied and implemented? Given the distinction between the nature of research produced by developed countries (primarily science-led) and developing countries (primarily problem-led), it would appear that a division of labor already exists.

In this context, perhaps a more important question is to what degree does information exchange exist between researchers from developed and developing countries? This is particularly important in the context of the present study, given that one of the first steps in attempting to identify 
possible methods to prevent and control such diseases is to analyze and understand the causes.

However, information exchange presupposes that the science (knowledge) base in all developing countries where these diseases are endemic is such that the results of fundamental biomedical research can be assimilated and used.

This again underlines the need to inventory and assess the amount and nature of (national) expertise currently available.

2. If the purpose of health research is to generate knowledge in order to improve health, should all such research be more in tune with national health needs than the forces that drive the research agendas of the international scientific community?

And where does this leave decision makers (especially in developing countries) who, in allocating scarce resources, must have at their disposal predictions about the potential for the success and usefulness of health research results? For example, with respect to the three tropical diseases considered here, does this mean that related health research-from the perspective both of the public and the government, must have practical applications? Of course, while this may be valuable for the medical community, it will not necessarily be a priority for biomedical researchers, whose primary interest may be the advancement of scientific knowledge. This raises the question of the relationship between different types of health and health research priorities.

Important in the complex decision-making process involving these two questions is to clearly identify desired outcomes or objectives, relevant actors and resources (human, financial, and physical) (Saaty, 1980:ix). ${ }^{9}$ For example, where the objective is the contribution of a given component, e.g., biomedical research, to overall health, it is important to understand the role and structure of every other component (for example, R\&D (medical and pharmacological), training (medical and research), primary health care, delivery and insurance systems, socioeconomic and environmental factors). In addition, consideration must be given to the vested interests of the various actors: donor governments and related agencies; developed and developing country researchers; policymakers, the medical community, and patients (and public opinion) in the various developing countries.

In the present context, this means that in dealing with the issue of health and health research priorities, decision makers (in developing and donor countries) could make greater use of different multispecialty expert groups to determine and assess whether (bio)medical research is worth the cost, compared to the alternative of implementing socioeconomic measures. This would involve, for example, comparing the feasibility and potential effects of improving rural housing, or altering irrigation systems and practices against large $R \& D$ investments in vector control, vaccine development, etc. Important here is that the value of, for example, a new vaccine, must be estimated in conjunction with the existence, or potential development of delivery methods and infrastructures to apply such (bio)medical tools (W.H.O., 1977; 1993a). In short, the methods chosen must be appropriate and applicable. 
Moreover, in this senario, it would be possible to compare the cost-effectiveness and feasibility of a socioeconomic, as opposed to a (bio)medical approach to fighting a given aspect of a tropical disease (in contrast to the problems associated with applying cost-benefit analysis to the general issue of health care, or the case of linking specific expenditures in biomedical research with health outcomes).

This is especially so where the choice is between a socioeconomic approach of potential use for providing criteria on which to base decisions on resource allocation for the control of tropical diseases, or for improving the application of existing control measures, or as an aid in making choices about, and the subsequent application of new tools; where a heavy investment in a particular area of biomedical research is associated with a high degree of risk and uncertainty (W.H.O., 1977; 1993a). ${ }^{10}$

Of course, this is not to imply that fundamental biomedical research should not constitute a priority in developing countries. Rather, that setting such priorities be based on a valid assessment of available expertise and be contingent upon information exchange and communication between (all) relevant actors.

Quantitative information on the output of the health research community is a valuable first step in the complex process of improving the contribution health research makes to the solution of high priority health problems in developing countries. ${ }^{11}$

\section{Acknowledgments}

The authors would like to thank Prof. W. van Rossum, Faculty of Organization and Management, University of Groningen, for his critical review and useful commentary. We would also like to extend our thanks to Dr. R. Vos, and J. Hoek, University Center for Pharmacology for their assistance in providing the software programming using to conduct this study.

\section{Notes}

1. Although there are considerable limitations, currently available bibliometric techniques designed to measure reseanch quality and productivity can increasingly be applied to selected areas of developing country output.

Important here is that advanced bibliometric techniques can also potentially be used to differentiate research productivity at the national and international levels in order to identify which aspects of existing research capacity need to be strengthened, and to generate an appropriate basis for national policy analysis. Such analyses can help to identify active researchers, distinguish between local and international research, and indicate the dissemination of research results (north/south, south/north, south/ south). As such, the proper application of bibliometric evaluations can also be used as background material for research policy work (Thulstrup, 1992:29).

2. Ideally, of course, it remains that setting research priorities implies a preferred order of implementation of action. And, as Sayers has so aptly pointed out,

Policy should also establish the principles on which priorities are set, but it is not helpful to attempt to decide policy on priorities, even in the broadest terms, without consideration of what is possible and practicable; "a" priority is not necessarily the same as "the" priority. The former implies a recognised degree of urgency and desirability; the latter constitutes the result of the consideration of how resources should be allocated in order to maximise the cost-benefit achievement of the policy decision (Sayers, 1992:23). 
Nevertheless, it would be a mistake to perceive of such areas as biomedical, epidemiological and public health research, the relationship between health and environment, health systems research, and health technologies as being mutually exclusive. Indeed they are not. For example, while research on infectious and parasitic diseases invariably involves vaccine development, drug treatment, control and management, etc., the actual prevention of such diseases is a socio-political issue. This involves national social and health policy development designed to effect urgently required improvements in the social (to include health care) and physical environment of the population.

3. See, for example, Walsh, J.A. (1989). Disease Problems in the Third World. In Bloom, B.R. and Cerami, A. (Eds.). Biomedical science and the third world. New York: New York Academy of Sciences (Vol. 569), pp. 1-16; Lopez, A.D. (1990). Causes of death: An assessment of global patterns of mortalitity around 1985. World Health Statistics Quarter$l y, 43$ (2):91-104.

4. As a result, instead of measuring the output (quantity and quality) of research communities in developing countries, donor organizations have opted to list research input measures (including such data as the number of per capita researchers; research expenditures in percentage GDP) (Thulstrup, 1992:14). Worse, such lists have been used as the basis for international comparisons.

More recently, however, at least some donor organizations (such as the World Bank) have become aware of the need to use currently available techniques to attempt to generate a systematic inventory and assessment of the quantity and quality of existing research activity in individual developing countries (Thulstrup, 1992:29).

5. Until 1984, EMBASE was the most frequently used international medical data base. It was superseded by MedLine, which includes more countries and non-English language publications.

MedLine is a computerized data base of medical sciences, containing medical subject headings based on the U.S. National Library of Medicine (which also produces this database).

It is available in three forms: published, on-line, and CD-ROM. The latter two correspond to the three printed bibliographies: Index Medicus, Index to Dental Literature, and International Nursing Index. These bibliographies include primarily journal references; approximately 3,600 international journals are regularly and systematically screened for this purpose.

MedLine outputs include the following information per record (article): title (English and original); author(s) in order of first, second, etc.; address (affiliation); journal in which article is published; year of publication; language of article; national affiliation of journal; whether the article comprises a letter, journal article, commentary, etc.

6. A journal was considered "regional" if the name of a southern or non-prominent region or country appeared in the title. Otherwise it was qualified as "international." A journal was defined "specific" if the names of diseases or the word "tropic" appeared in the title.

7. Countries that have at least 1\% of total output in 1989, 1990 or 1991 (not rank ordered): Malaria:

Developed: United States of America, United Kingdom, France, Australia, Switzerland, Germany, Italy, Sweden, Canada, Japan, Netherlands, Israel.

Developing: India, Thailand, Nigeria, China, Gambia, Kenya, Cameroun, Papua New Guinea, Ceylon, Indonesia, Malaysia, Tanzania, Madagascar.

Schistosomiasis:

Developed: Australia, Canada, Denmark, France, Germany, Israel, Italy, Japan, Netherlands, South Africa, Sweden, Switzerland, UK, US, Spain.

Developing: Kuwait, Brazil, China, Egypt, Kenya, Nigeria, Saudi Arabia, Sudan, Zambia, Sri Lanka, Zimbabwe, Tanzania

Leprosy:

Developed: Australia, France, Germany, Japan, Netherlands, Portugal, UK, US, Belgium, Canada.

Developing: Brazil, Ethiopia, India, Mexico, Senegal, Thailand, China, Nepal, Turkey. 
8. A number of reasons are usually given to explain this phenomenon, some of the most important of which include the shortage or lack of expert counterparts and facilities in developing countries; the lack of incentive on the part of counterparts; the pressures of producing high quality research either discourages the engagement of counterparts, or places constraints on their participating fully in the research endeavor; inadequate donor funding incentives; it is easier for developed country researchers to do the work at home and then field test it (thus engaging counterpart institutions but not counterpart researchers).

9. Saaty has identified twelve primary decision-making concerns: planning; generating a set of alternatives; setting priorities; choosing the best policy after finding a set of alternatives; allocating resources; determining requirements; predicting outcomes; designing systems; measuring performance; insuring the stability of a system; optimizing; and resolving conflict (Saaty, 1980:5).

10. Of course, there are also many problems associated with the planning and implementation of relevant social science research. For example, such research is variably dependent on the socioeconomic and ecological conditions at the regional, national, and local levels (in addition to the distinction between the urban and rural setting); it requires a multidisciplinary approach, in order to inventory and assess the socioeconomic dimensions of such diseases; related research activity will have to be planned and conducted from within the development (agriculture, irrigation, etc.), rather than the medical or health context; social science research on tropical disease control and prevention involves close coordination with epidemiological research activities (which often are negligible to nonexistent); such research must be conducted in the context of policy action requirements, i.e., it must be problem, rather than science led, and must generate short or medium-term practical results (W.H.O., 1977; 1993a).

11. Such information can also be used to identify those actors (researchers and institutions) in developing countries, producing relevant "grey" (or fugitive) literature (i.e., final reports, working papers, monographs, and so on), not normally appearing in available data bases.

\section{References}

Christian, M. (1988). A Guide to Leprosy Control. 2d ed. Geneva: W.H.O.

Essential National Health Research (ENHR). (1991). A Strategy for Action in Health and Human Development. Produced by the Task Force on Health Research for Development. Geneva, Switzerland: United Nations Development Programme (UNDP).

Gaillard, J. (1990). Science in the developing world: Foreign aid and national policies at a crossroad. Ambio, 19(8), pp. 348-353.

Girdwood, A. (1991). Capacity Building and African Higher Education. In, conference proceedings on "Strengthening Analytical and Research Capacities in Education: Lessons from National and Donor Experience." W. Gmelin and K. King (Eds.). Bonn: ZED

GPA (Global Programme on AIDS) (1992). "Priorities for Research." Prepared by the Steering Committees of the GPA, revision 3, November.

Health Systems Research (HSR). (Update, 1992). Health systems research: Does it make a difference? The joint HSR project in the Southern African region. Produced in conjunction with W.H.O., Netherlands Ministry for Development Cooperation and the Royal Tropical Institute.

International Development Research Centre (IDRC). (1992). Health and the environment: A people-centred research strategy. Ottawa, Canada: IDRC.

Krasno, R. (1979). The foundation's international education program: A review of issues and options for the future. International Division Education Conference, 2-4 October, New York: Ford Foundation.

Lopez, A.D. (1990). Causes of death: An assessment of global patterns of mortality around 1985. World Health Statistics Quarterly, 43 (2):91-104.

Maurice, J. \& Pearce, A.M. (Eds.). (1987). Tropical disease research: A global partnership: UNDP, World Bank, W.H.O. Special programme for research and training in tropical diseases. Geneva: W.H.O. 
RAWOO (Advisory Council for Scientific Research in Development Problems). (1984). Report on health and illness in developing countries: Research needs and priorities. The Hague, The Netherlands: RAWOO.

Saaty, T.L. (1980). The analytical hieranchy process: Planning, priority setting, resource allocation. New York: McGraw-Hill.

Sayers, B. McA. (1992). Health policies, priorities and research. In Davies, A.M. and B.P. Mansourian (Eds.), Research strategies for health. Lewiston, N.Y.: Hogrefe \& Huber, pp. 22-30.

Thulstrup, E.W. (1992). Improving the quality of research in develping country universities. PHREE Background Paper Series, Doc. No. PHREE/92/52. Washington DC: The World Bank.

Van Raan, A.F.J. (1993). Advanced Bibliometric Methods to Assess Research Performance and Scientific Development. Review paper presented at the International Conference on Evaluation, Social Science and Public Policy, Ottawa, Canada, 9-11 June 1993.

Walsh, J.A. (1989). Disease problems in the Third World. In Bloom, B.R. and Cerami, A. (Eds.). Biomedical science and the Third World. New York: New York Academy of Sciences (Vol. 569), pp. 1-16.

W.H.O. (1977). Special programme for research and training in tropical diseases. Annual Report. Geneva: W.H.O.

W.H.O. (1987). Tropical disease research: A global partnership. Eighth programme report of the UNDP/World Bank/W.H.O. Special programme for research and training in tropical diseases (TDR). Geneva: W.H.O.

W.H.O. (1988). Expert committee on leprosy: First report. Technical report series. Geneva: W.H.O.

W.H.O. (1990). Global estimates for health situation assessment and projections. Division of epidemiological surveillance and health situation and trend assessment. Geneva: W.H.O.

W.H.O. (1992). Our planet, our health: Report of the W.H.O. Commission on Health and Environment. Geneva, Switzerland: W.H.O.

W.H.O. (1993). The control of schistosomiasis: Second report of a W.H.O. expert committee. WHO technical report series. Geneva: W.H.O.

W.H.O. (1993a). Tropical disease research: Progress 1991-92. Eleventh programme report of the UNDP/World Bank/W.H.O. special programme for research and training in tropical diseases (TDR). Geneva: W.H.O.

Williams, A.P., Carter, G.M., Harman, A.J., Keeler, E.B., Manning Jr., W.G., Neu, C.R., Pearce, M.L. \& Rettig, R.A. (1976). Policy analysis for federal biomedical research. Prepared for the president's biomedical research panel. R-1945-PBRP/RC. Santa Monica, CA: Rand. 